\title{
Response Adaptive Modelling for Reducing the Storage and Computation of RSS-based VLP
}

\author{
Sander Bastiaens, David Plets, Luc Martens and Wout Joseph \\ INTEC - WAVES, Ghent University/imec, Ghent, Belgium \\ Email: Sander.Bastiaens@UGent.be
}

\begin{abstract}
The precise (location) tracking of automated guided vehicles will be key in enlarging the productivity, efficiency and safety in the connected warehouse or production infrastructure. Combining the modest price tag, the adequate coverage and the potential centimetre accuracy makes Visible Light Positioning (VLP) systems appealing as replacements for the current, high-cost, tracking systems. Model-fingerprinting-based received signal strength (RSS) VLP enables the required accuracy. It requires an elaborate optical channel model fingerprinted in a fine-grained, and predefined positioning grid. Depending on the grid's granularity, constructing the fingerprint database demands a significant computation and storage effort. This paper employs response adaptive or sequential experimental design to form sparse channel models, vastly reducing the storage and computation. It is shown that model-fingerprinting-based RSS only requires modelling less than 1 percent of the grid points, in an elementary positioning cell. The sparse model can be re-evaluated as a way to cope with environment changeover. Model recomputation as a way of compensating for LED ageing is also studied.
\end{abstract}

Keywords - Visible Light Positioning, VLP, RSS, fingerprint, storage, Response Adaptive, Sequential Experimental Design

\section{INTRODUCTION}

To improve production processes in terms of efficiency, safety and environmental impact, several Governing Bodies target using more automation and Internet of Things (IoT) paradigms in industry, production and logistics. The European Commission for example launched several initiatives with this objective, such as Horizon 2020, Industry 4.0 and SmartFactory. The goal is to form connected warehouses or connected industry infrastructures, where a growing population of robots and Automated Guided Vehicles (AGVs) should help enlarging the productivity and help minimizing operating costs.

Consequently, market research companies perceive and even forecast an increased demand for using automated guided vehicles (AGVs) [1]. As of 2016, the AGV market was already worth an estimated (USD) $\$ 1.12$ billion [1]. Precisely (location) tracking, efficiently and dynamically managing (routing) automated transportation means is of uttermost importance in increasing the overall efficiency of a value chain [2].

Currently, AGVs are guided via slotted wires in the floor (difficult to dynamically adapt the routes), via guide tape (which can be damaged, dirtied and does not allow reaching arbitrary spaces), via costly pulsed or modulated lasers, via
RF-based solutions (limited accuracy), or via vision guidance (large power consumption) [3]. Unfortunately, these approaches seem to (at present) suffer from at least one significant issue. Alternatively, the use of visible light for location tracking i.e. Visible Light Positioning (VLP) may empower low-cost AGVs, self-driving along any route.

Research has already demonstrated VLP's viability, see e.g. the studies of Zhou [4] and the localisation systems: Epsilon (beaconing) [5], Luxapose (rolling shutter effect) [6], Lightitude (received light strength model) [7] and others (more information on VLP can be found in designated surveys [8]). Published works proved that VLP can theoretically (either based on the mathematical derivation of the Cramér-Rao lower bound or based on simulations) provide an accuracy in the order of centimetres. Some practical systems have shown decimetre order accuracy, but these positioning resolutions are usually obtained in idealised environments (e.g. at limited receiver-LED distance) [8], [9]. Yet, the combination of the limited cost (in terms of installation, changeover and environmental impact), adequate coverage (due to the solid state lighting revolution) and the potential centimetre accuracy of VLP systems [8], [9], does make them highly appealing as replacements for the current, high-cost, tracking systems.

To obtain accurate 2D localisation of an AGV operating in an industrial hall corner, this paper employs modelfingerprinting-based received signal strength (RSS). The latter makes use of a precomputed propagation map, which accounts for the relevant channel characteristics and which continuously remains at the disposal of a photodiode (PD)-equipped receiver. The propagation model holds RSS values for each LED on all locations on a predefined positioning grid (e.g. with a location resolution of $1 \mathrm{~cm}$ ). The grid position which holds the closest match between modelled and measured RSS values is taken to be the receiver position. As opposed to the works in literature, this paper defines its RSS values in terms of the receiver photocurrent's individual LED contributions, to closer resemble a VLP system's real-life deployment. It consequently considers the angular dependence of the photodiode's responsivity. This angular dependence arises from Fresnel losses (i.e. the photodiode's surface reflectance changes with the incidence angle) or from the photodiode package itself [10], [11]. The extensive effort needed to either compute (as in e.g. [12]) or store the propagation map, renders the 
model-fingerprinting approach rather infeasible. Also, model recomputation in response to infrastructure changeover, failure (of e.g. a few LEDs) or performance decreasing factors is greatly hindered. To minimize model (re)generation or storage, this paper brings as novelty the response adaptive or sequential experimental design paradigm (SED) [13]-[15] to Visible Light Positioning. The SED principle allows greatly reducing the channel model computation and storage. Model recomputation as to compensate for LED ageing is also studied.

\section{BACKGROUND}

The domain of logistics/tracking and tracing leaves a promising potential application for VLP, provided that it can provide a highly accurate, robust and low cost solution. Received Signal Strength (RSS)-based visible light-based localisation minimizes the system requirements: a sole PD or image sensor suffices. RSS-based positioning estimates require elaborate propagation (i.e. optical channel) models for them to be accurate [9]. RSS-based VLP usually entails using either trilateration or (manual) fingerprinting. Manual fingerprinting brings accurate localisation with, but at the cost of substantial effort. Trilateration relates an RSS value $R S S_{i}$ per $\mathrm{LED}_{i}$ (generally the incident (radiant) power $P_{R, i}$ or the induced photocurrent $I_{P D, i}$ ) to the distance $d_{i}$ between the receiver's origin $\left(x_{U}, y_{U}, z_{U}\right)$ and each of the LEDs, before determining the absolute position. In a general form, $R S S_{i}$ cannot be expressed as an invertible function of $d_{i}$, due to the presence of reflections. Limiting the complexity of the $R S S_{i}-d_{i}$ relation does impede highly accurate positioning. Combining both positioning principles, coined model-fingerprinting-based RSS, should allow for more truthful positioning estimates [12]. The localisation consists of the 3 steps shown in Fig. 1.

\section{A. Model-fingerprinting-based RSS Localisation}

$K$ of the ubiquitously available LED fixtures are intensity modulated. This is performed in such a way that the individual LEDs' contributions are separable at the receiver. LEDs are mainly employed as they can be simultaneously used for positioning (and communication) as well as for efficient illumination purposes. In response to the incident light, the receiver photodiode (PD) generates a 'weak' photocurrent $I_{P D}$ that is proportional (assuming a linearly operating PD [6]) to the incident instantaneous radiant flux (i.e. 'optical power'). After being electronically processed (amplified, converted and filtered), $I_{P D}$ is decomposed into a photocurrent contribution per $\operatorname{LED}_{i} I_{P D, i} .\left\{I_{P D, i}\right\}$ are also taken to be the RSS values, used in the actual position estimation step (see Fig. 1). In the third step of the VLP localisation procedure, the receiver searches the closest match between modelled and observed RSS values. (Offline) elaborately modelling and discretising the propagation environment, allows precomputing those modelled RSS values (i.e. forming a map of fingerprint values per LED). Fingerprint matching is in essence an exhaustive algorithm. This paper does not tinker with the exhaustive search and uses the root-mean-square error (rMSE) as matching cost function on the $\left\{I_{P D, i}\right\}$ set [12].
The industrial, producing or distributional hall may be a particularly challenging positioning environment. It may entail large (for VLP) transmitter-receiver distances, the presence of (highly) reflective surfaces, and the disparity in, and constraints of to be retrofitted LED fixtures (the LEDs may be tilted or even time-worn). To account for the performanceimpacting channel factors [16], [17] and all relevant VLP system components (e.g. the LEDs' radiation patterns), modelfingerprint-based RSS requires extensive (and computationally expensive) channel models (though efficient modelling approaches have been published, e.g. the combined deterministic and modified Monte Carlo (CDMMC) channel model of $\mathrm{Gu}$ et al [18] or the analytical method of Chen et al. [19]). The propagation model holds RSS values for each LED on all locations on a predefined positioning grid. This grid is usually defined upfront (all samples selected at once) and is frequently uniform and rectangular. Depending on the predefined granularity (e.g. a resolution of $1 \mathrm{~cm}^{2}$ ) and the exploration space (e.g. an industrial hall with dimensions of $100 \mathrm{~m}$ by $100 \mathrm{~m}$ ), the grid requires (in complete form) significant storage e.g. $10^{8} \cdot K$ values, which with a precision of 8 bytes results in approximately $298 \mathrm{~GB}$ of required storage for 400 LEDs.

\section{B. Response adaptive modelling}

The previous paragraph described the necessity for sparse channel models in model-fingerprinting-based RSS. To introduce sparseness in RSS fingerprints, Liu et al. [20] employ 'ordinary' kriging (i.e. spatial interpolation) to estimate the RSS values at arbitrary locations given existing fingerprints. The kriging procedure makes use of an empirical variogram, a (fitted) mathematical model to interpolate. In VLP use cases, in particular in highly reflective environments (i.e. with (arbitrary) obstacles introducing a significant Non-Lineof-Sight (NLOS) contribution), the empirical variogram may prove difficult to fit. An improper variogram will subsequently introduce significant channel model and positioning errors. SparseLoc [21] introduced the Orthogonal Matching Pursuit $(O M P)$ algorithm (for WIFI), which compresses an RSS vector per location by representing it with respect to a linear combination of a few base signals (forming a sparse dictionary).

For both techniques, a predefined number of samples are selected up front (in one take), before running the sparse modelling. This one-shot selection can be prone to both oversampling and undersampling [15]. Sequential Design (SED) allows the sequential (i.e. iterative) generation of a spacefilling design. The sample collection's outputs (here: the optical channel gain) can be used for a next design iteration by building a global Surrogate Model [15] (an approximation function). Aerts et al. combined SED and surrogate modelling for charting the RF-EMF exposure in urban areas [22].

Highly efficient space-filling SED algorithms can easily be wielded by employing the Sequential Experimental Design (SED) (MATLAB) Toolbox of Crombecq et al. [13], [14]. This paper considers two algorithms, denoted LOLA-Voronoi [13] and $m c$-intersite-proj-th [14]. Mc-intersite-proj-th is a Monte Carlo sampling method that evaluates a criterium (based on 


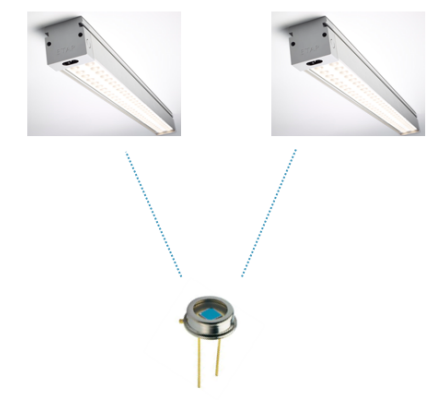

Photocurrent (RSS) measurements

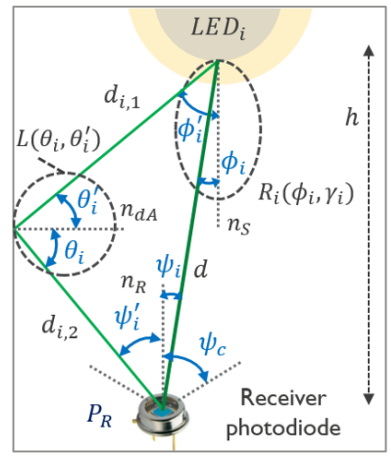

Optical Channel Model

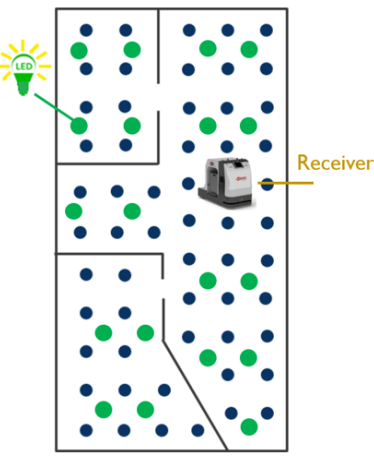

Fingerprint matching

Fig. 1. The model-fingerprinting-based RSS principle: measuring the RSS values, forming a channel model (creating the fingerprint) and fingerprint matching

the intersite (maximin) and projected distance) for the random candidate points. The LOLA-Voronoi algorithm couples Monte Carlo-based Voronoi tessellation (exploration) and local linear approximations of the associated sample output (exploitation).

Details and mathematical analysis of the SED algorithms can be found in [13]-[15]. The SED approaches can be combined with other sparse modelling approaches i.a. Gaussian Mixture Modelling (only store the Gaussian parameters), Kriging [20] or sparse dictionaries [21].

\section{SYSTEM DESCRIPTION}

In this paper, all simulations are performed, and all models are built for an AGV localisation use case. An AGV operates in the corner part of what could be either an industrial, manufacturing or distribution hall. The propagation room is depicted in Fig. 2. This section discusses respectively the LED transmitter infrastructure (in section III-A), the receiver VLP setup and the optical channel (in section III-B).

\section{A. LED infrastructure}

$K=6$ incoherent white chip-on-board (COB) LEDs are pre-empted for VLP purposes. The LEDs, designated $\mathrm{LED}_{i}, i=1 . . K$, are hung from the ceiling at preset positions $\left(x_{S, i}, y_{S, i}, h\right), i=1 . . K, h=6$. The LEDs are intensity modulated as to transmit signals, whose contributions to the received photocurrent $I_{P D}$ are demultiplexable at the receiver (using appropriate modulation techniques, see e.g. [23], [24]). Both the LED coordinates and all needed demultiplexing information is assumed to be provided to the receiver. As in industry the LED-to-PD distance is generally amply large $(>5 \mathrm{~m}), \mathrm{LED}_{i}$ are assumed modellable as Lambertian radiating point sources (of order $m_{i}=1$ ). Each LED radiates an early-lifetime optical power (i.e. a radiant flux amplitude) $P_{t, i}(0)=P_{t, i}$. Over time, the LEDs' luminous intensity will dwindle i.e. LED ageing. As opposed to the sudden failure behaviour of incandescent illumination, LED lighting's radiant flux degrades gradually over its (longer) operating time [25]. In this paper, the influence of LED ageing is modelled as an exponential decay on the nominal transmitted flux $P_{t, i}$ [26]:

$$
P_{t, i}(t)=P_{t, i}(0) \cdot \exp (-a t), a=\frac{-\ln (0.7)}{t_{o p}}
$$

where $t_{o p}$ and $t$ represent the $70 \%$ lumen maintenance operating time (L70) and the LED's current lifetime (in hours) respectively. (1) makes abstraction of the impact (e.g. in spectrum) of each light loss factor (e.g. thermal effects) [27].

\section{B. Receiver and Environment}

Each AGV is equipped with a single circular photodiode (PD) (with active area $A_{R}=1 \mathrm{~cm}^{2}$ and field of view $\psi_{C}=$ $70^{\circ}$ ), located $h_{r e c}=1 \mathrm{~m}$ above the ground plane. Knowing the height of the AGV, the localisation problem reduces to a 2D one. The centre position of the AGV coincides with the PD's centre. Assuming an optical channel that is both timeinvariant stationary and having a flat frequency characteristic (over the operating range) and given the transmitted flux $P_{t, i}(t)$, the generated photocurrent $I_{P D}$ satisfies:

$$
I_{P D}(t)=\sum_{i=1 . . K} P_{t, i}(t) h_{c, P D}^{(i)}, h_{c, P D}^{(i)}=R_{p}\left(\psi_{i}\right) \cdot h_{c}^{(i)}
$$

$h_{c, P D}^{(i)}$ denotes the DC channel gain of the propagation from $\mathrm{LED}_{i}$, and is used to estimate the receiver location (section II-A). The receiver's axially symmetric (angular) responsivity $R_{p}\left(\psi_{i}\right)\left(R_{p}\left(\psi_{i}\right)=0,\left|\psi_{i}\right|>\psi_{C}\right)$ relates $h_{c, P D}^{(i)}$ to the DC channel gain on radiant powers $h_{c}^{(i)}\left(\psi_{i}\right.$ designates the incidence angle from $\operatorname{LED}_{i}$ ):

$$
R_{p}\left(\psi_{i}\right)=R_{P}\left[1-\left(\frac{\psi_{i}}{\psi_{C}}\right)^{2}\right],\left|\psi_{i}\right| \leq \psi_{C}
$$

Similarly as in most VLP related papers (Uysal et al. proposed alternative visible light models in [28]), $h_{c}^{(i)}$ is modelled in terms of a Line-of-Sight $(L O S) h_{c, L O S}^{(i)}\left(h_{c, L O S}^{(i)}=0,\left|\psi_{i}\right|>\right.$ $\left.\psi_{C}\right)$ and Non-Line-of-Sight (NLOS) components $h_{c, N L O S}^{(i, d A)}$ $\left(h_{c, N L O S}^{(i, d A)}=0,\left|\psi_{i}^{\prime}\right|>\psi_{C}\right): h_{c}^{(i)}=h_{c, L O S}^{(i)}+\sum_{A} h_{c, N L O S}^{(i, d A)}$ Both are expressed using the infrared (IR) light models proposed by Kahn et al. [29]. The filter and concentrator gain are set to 1 [29]. $\mathrm{LED}_{i}$ 's radiation pattern $R_{i}\left(\phi_{i}, \gamma_{i}\right)$ reduces for a Lambertian radiator to: $R_{i}\left(\phi_{i}, \gamma_{i}\right)=R_{E}\left(\phi_{i}\right)=\left[\frac{m+1}{2 \pi} \cos ^{m}\left(\phi_{i}\right)\right]_{m=1}$. $\gamma_{i}$ is the azimuth angle in the LED plane. $h_{c, N L O S}^{(i, d A)}$ results from reflections on walls and obstacles. It is modelled in terms of (ideally infinitesimally) small surface elements $A$ (with 


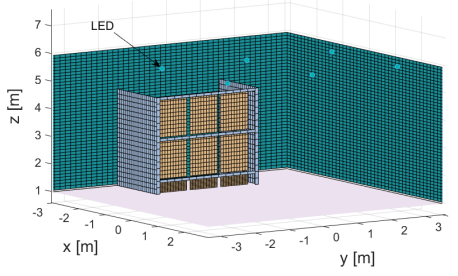

(a)

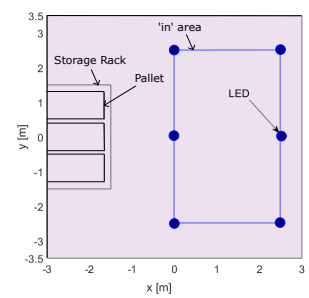

(b)
Fig. 2. The side (a) and aerial view (b) of the VLP simulation room. The discretisations of the reflectors are shown. $\left\{\mathrm{LED}_{i}\right\}$ are indicated.

area $d A$ ). To limit computations, the subsequent analysis only accounts for $1^{\text {st }}$ order reflections.

$$
\begin{gathered}
h_{c, L O S}^{(i)}=R_{i}\left(\phi_{i}, \gamma_{i}\right) \cdot \frac{A_{R} \cdot \cos \left(\psi_{i}\right)}{d_{i}^{2}} \\
h_{c, N L O S}^{(i, d A)}=R_{E}\left(\phi_{i}^{\prime}\right) \cdot \frac{d A \cdot \cos \left(\theta^{\prime}\right)}{d_{i, 1}^{2}} \cdot \frac{A_{R} \cdot \cos \left(\psi^{\prime}\right)}{d_{i, 2}^{2}} \cdot L\left(\theta_{i}, \theta_{i}^{\prime}\right)
\end{gathered}
$$

$d_{i}, \phi_{i}, \phi_{i}^{\prime}$ and $\theta_{i}$ represent the $\mathrm{LED}_{i}$-to-PD distance, the irradiance angle at the LED for the LOS and NLOS component, and the irradiance angle during reflection respectively. $\theta_{i}^{\prime}$ and $\psi_{i}^{\prime}$ cover the incidence angle at respectively the wall and PD. Fig. 1b. shows the definition of the quantities above.

$L\left(\theta_{i}, \theta_{i}^{\prime}\right)$ models the reflected radiation pattern as consisting of both a diffuse and specular part i.e. according to Phong's model [30]. (6) renders Phong's model with $\rho, r_{d}, m_{d}, m_{s}$ being the reflection coefficient of the surface, the fraction of diffusivity, and the Lambertian orders of the diffuse and specular component.

\section{Simulation Configuration}

All simulations are performed inside the room, depicted in Fig. 2. The AGV's area of activity measures $6 \mathrm{~m}$ by $7 \mathrm{~m}$ and is enclosed by a north and west uniform (grey Portland cementbased [31]) concrete wall (with $m_{d}=1, r_{d}=1, \rho=0.4$ [30]). The room is uniformly meshed with a grid point every $2.5 \mathrm{~cm}$ (i.e. unless explicitly specified). Part of the west wall is covered with a metallic storage rack $\left(m_{d}=1, m_{s}=6\right.$, $\left.r_{d}=0.2, \rho=0.85\right)$ of $1.5 \mathrm{~m}$ width and $4.5 \mathrm{~m}$ height. The rack, which has a frame thickness of $10 \mathrm{~cm}$, stretches over $3 \mathrm{~m}$ and harbours 3 shelves each with 3 pallet stacks. Each stack consists of 10 wooden pallets $\left(m_{d}=1, r_{d}=1, \rho=0.2\right)$ of dimensions $1.2 \mathrm{~m}$ x $0.8 \mathrm{~m}$ x $14.4 \mathrm{~cm}$. The walls, the rack's side panels, the rack's frame and the pallets are per dimension discretised into 45, 20, 15 and 10 surface elements.

The reference coordinate system $\mathrm{X}-\mathrm{Y}$ is located at the floor's centre. The inter-LED distance is set to $2.5 \mathrm{~m}$. The LEDs, whose coordinates are $([\{0,2.5\},\{-2.5,0,2.5\}, 6])$, are fabricated to transmit a nominal $P_{t, i}$ of $5 \mathrm{~W}$. The receiver photodiode, characterised by a maximum responsivity $R_{P}$ of $0.5 \mathrm{~A} / \mathrm{W}$, exhibits noisy behaviour. The noise influence is modelled in terms of an input-referred current noise, which is assumed to be both additive and Gaussian with a zero mean and a $\sigma^{2}$ variance (a noise component is added to (2)).

The localisation accuracy is consistently evaluated in terms of the rMSE between the estimated $\left(x_{D}, y_{D}, h_{r e c}\right)$ and $\left(\theta_{i}, \theta_{i}^{\prime}\right)=\rho\left[r_{d}\left(\frac{m_{d}+1}{2 \pi}\right) \cos ^{m_{d}}\left(\theta_{i}\right)+\left(1-r_{d}\right)\left(\frac{m_{s}+1}{2 \pi}\right) \cos ^{m_{s}}\left(\theta_{i}^{\prime}-\theta_{i}\right)\right]$

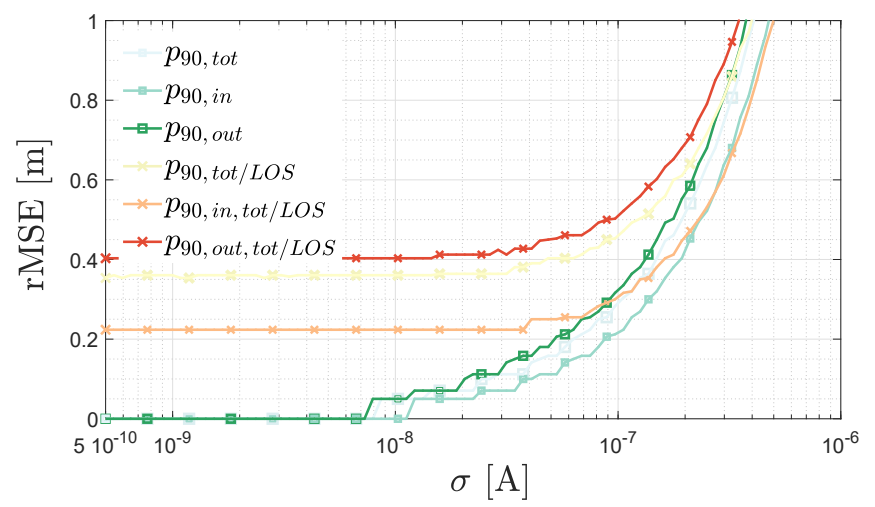

Fig. 3. The influence of input-referred current $\mathrm{rms}$ noise $\sigma$ on the positioning accuracy (rMSE) $p_{90}$ for the uniform grid

the actual $\left(x_{U}, y_{U}, h_{r e c}\right)$ position, defined as: $r M S E=$ $\sqrt{\left(x_{D}-x_{U}\right)^{2}+\left(y_{D}-y_{U}\right)^{2}}$ in $[\mathrm{m}] . p_{90}$ denotes the $90^{\text {th }}$ percentile rMSE. The considered sparse modelling techniques can be applied in complete industrial halls. To limit the computational effort, it is assumed that the AGV's operating area is obtainable from a complete model (e.g. by being an elementary positioning cell which compose the industrial hall).

\section{Sparse Model Evaluation}

An optical channel that closely resembles real-life propagation is vital to ensure highly-accurate positioning estimates (i.e. step 2 of the localisation approach as depicted in Fig. 1). As stated in section II, a practical model-fingerprinting-based localisation system requires curtailing the computation, storage and matching procedure. Furthermore, due to i.a. infrastructure changeover or (partial) failure, the channel model may need to be updated as to ensure a reasonable positioning performance.

The requirement of minimizing the latency/downtime and storage advocates the use of a sparse representation of the optical channel. This section evaluates the use of non-gridbased Sequential Experimental Design (see section II-B).

Section IV-B identifies the response model (i.e. the output) best used in the sequential design, i.e. both for the mc-intersiteproj-th and LOLA-Voronoi algorithm. It also indicates the level of sparseness that can be introduced, whilst maintaining the capability to well-approximate the channel over a complete uniform grid. Well-approximating means not heavily compromising the positioning performance. Section IV-A provides the baseline, with which to compare the sparse modelling performance. It discusses the extent of the influence of input-referred current $r m s$ noise $\sigma$ on the positioning accuracy. Section IV-B also compares sparsely modelling the optical channel with the expensive (uniform grid) baseline model. Section IV-C focusses on the actual application of the sparse models. To 


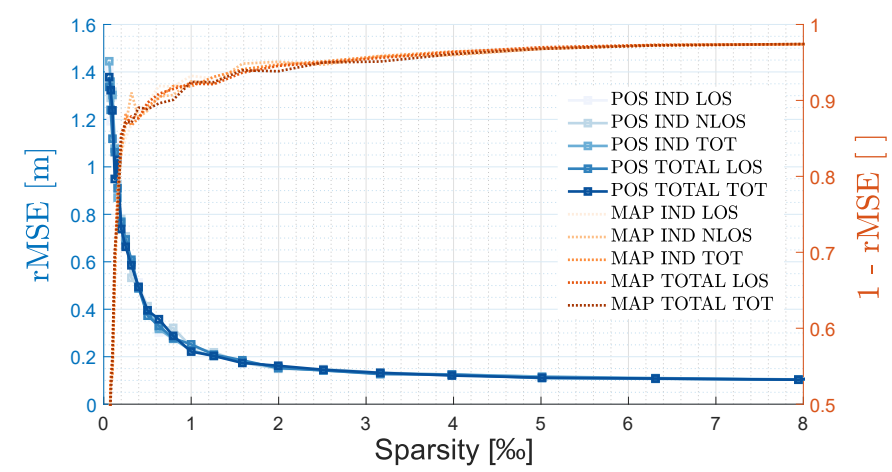

Fig. 4. The influence of sparseness on the (reconstructed) channel model 'MAP' (1-rMSE $M A P$ in red shades) and the positioning accuracy 'POS' ( $r M S E_{P O S}$ for $\sigma=10 \mathrm{nA}$ in blue shades) for $m c$-intersite-proj-th

combat ageing, the sparse representation is employed to (more) quickly recompute a propagation model update.

\section{A. Positioning on a Uniform Grid}

Before the adequacy of sparse models can be quantified, this part first discusses the influence of $\sigma$ on the positioning performance for every grid point ('tot'). Fig. 3 plots $p_{90}$ for a varying $\sigma$. The 'in' and 'out' subscript indicate that the $p_{90}$ calculation is limited to the area confined between ('in') (Fig. 2b) and outside ('out') the LEDs. Evidently, inside the area confined between the LEDs, $p_{90}$ is lowest. The $\left\{I_{P D, i}\right\}$ there maximally exceed the noise floor. At low $\sigma(\sigma \leq 5 \mathrm{nA})$ and thus high signal-to-noise-ratio (SNR), the $p_{90}$ is stable. Afterwards, an increasing $\sigma$ effectuates a dramatic increase in $p_{90}$. For $\sigma \leq 20 \mathrm{nA}, p_{90}$ satisfies the decimetre order accuracy constraint frequently used in literature. For $\sigma=10 \mathrm{nA}$, a representative number for a limited bandwidth junction gate field-effect transistor (JFET)-based transimpedance amplifier, $p_{90}$ equals $5 \mathrm{~cm}$. Fig. 3 also shows the resulting $\sigma-p_{90}$ relation when reflections are not accounted for in the modelled RSS values (subscript tot/LOS). It can be concluded that modelling reflections is important, as not modelling them introduces a significant $p_{90}$ offset (i.e. to well above $10 \mathrm{~cm}$ ).

\section{B. Identification of the Appropriate Sparse Model}

This section applies both the LOLA-Voronoi and the $m c$ intersite-proj-th algorithm to form sparse representations of the optical propagation model (i.e. in terms of photocurrent contributions). Each iteration, both Monte Carlo-sample generation methods output new samples, continuously distributed in the positioning area depicted in Fig. 2. The samples are barred from non-physical locations (no samples are generated where the storage rack is found). Per sample, the associated photocurrent magnitudes $\left\{I_{P D}, i\right\}$ are computed. The collection of samples and photocurrents yields the sparse model.

Mc-intersite-proj-th selects samples based on maximizing the projected distance. It is a purely space-filling approach. LOLA-Voronoi on the other hand reckons with the nonlinearity of the function used to optimise the sampling process i.e. the model function. In LOLA-Voronoi, candidate samples

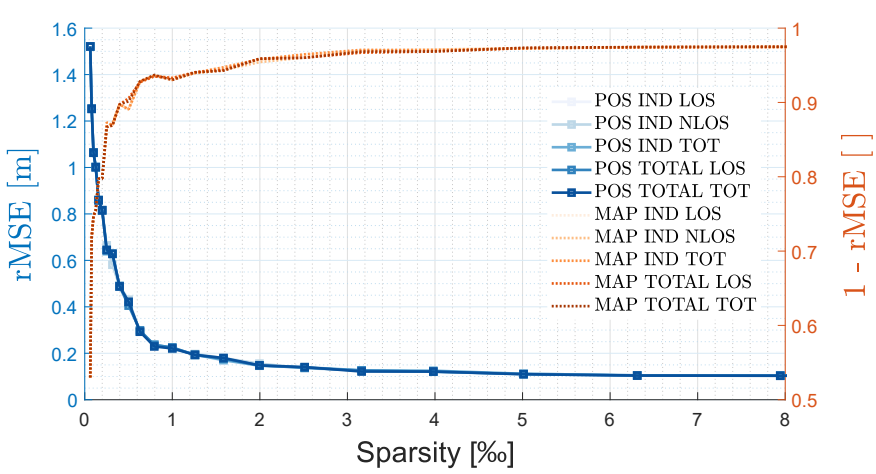

Fig. 5. The influence of sparseness on the (reconstructed) channel model 'MAP' (1-rMSE $M A P$ in red shades) and the positioning accuracy 'POS' ( $r M S E_{P O S}$ for $\sigma=10 \mathrm{nA}$ in blue shades) for LOLA-Voronoi

found in the non-linear regions receive a more pronounced consideration in the candidate sample selection process.

The model function or sequential design is per iteration updated by incorporating the newly generated samples and their appertaining outputs (albeit/albethey only effectively fed back and used for LOLA-Voronoi). The nature of the outputs is dictated by an output configuration, and associated output function. The model function will closer and closer resemble the output function as more samples are added. This paper considers 5 output configurations: 'IND LOS', 'IND NLOS', 'IND TOT', 'TOTAL LOS', 'TOTAL TOT'. Each configuration is denoted by two parts. The first part indicates whether the individual ('IND') photocurrent contributions of each LED, or whether the sum of the photocurrent contributions (i.e. the total photocurrent 'TOTAL'), is used to perform sample selection with. The second part denotes if the LOS, NLOS or the sum of both ('TOT') is employed as an optimization (output) function. The former 3 configurations thus entail an optimization procedure in $K$ (the number of LEDs) dimensions during the sample selection process, the latter 2 in 1.

Besides selecting the to be optimised output function, this section also indicates the level of sparseness that can be introduced before the positioning performance is heavily compromised. Hereto, both the rMSE on the modelled photocurrents and on the positioning are plotted for a varying number of generated samples (to build the channel model with).

Evaluating which of the sequential design algorithms is best used for this particular VLP use case, is a twofold procedure. First, the sparse model is interpolated to cover the uniform grid (this paper limits itself to bilinear interpolation). The $90^{\text {th }}$ percentile normalised rMSE value ' $r M S E_{M A P}$ ' (unitless) is computed to quantify the resemblance between the interpolated sparse model and the uniform grid model. In other words, the rMSE between modelled and measured photocurrent contributions $I_{P D, i}$ and $I_{P D, i}^{\prime}$ is normalised with respect to $I_{P D, i}$. Second, for an input-referred current $\mathrm{rms}$ noise magnitude $\sigma=10 \mathrm{nA}$, the $90^{\text {th }}$ percentile of the rMSE on the positioning ' $r M S E_{P O S}$ ' (in [m]) is also computed. Both $r M S E_{M A P}$ and $r M S E_{P O S}$ are computed for each point on the uniform grid (i.e. every $2.5 \mathrm{~cm}$ ). 


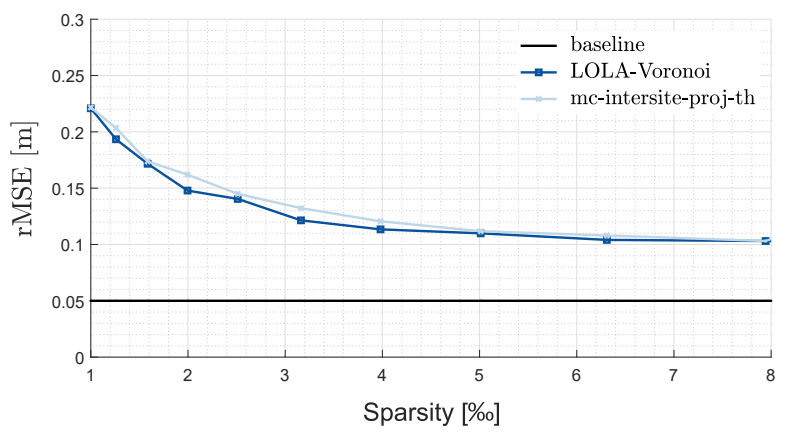

Fig. 6. Comparing the sparsity - $r M S E_{P O S}$ relation of LOLA-Voronoi and mc-intersite-proj-th for 'TOTAL TOT' with the uniform grid's baseline

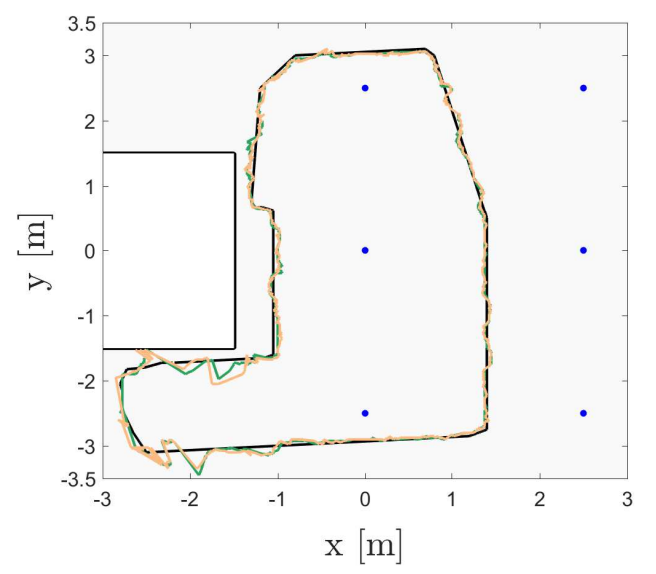

Fig. 7. Static positioning along a reference (in black) AGV trajectory for LOLA-Voronoi (in green) and mc-intersite-proj-th-based (in light orange) propagation maps

Figures 4 and 5 show $1-r M S E_{M A P}$ and $r M S E_{P O S}$ for the mc-intersite-proj-th and the LOLA-Voronoi algorithm respectively. Both $r M S E_{M A P}$ and $r M S E_{P O S}$ show exponentially decreasing trends in function of the number of generated samples, and this for both sequential design algorithms. When the number of samples contained in both sparse model exceeds 5 per mille ( $1 / 10$ of a percent) of the uniform grid, the performance gain becomes more gradual with an increasing amount of model samples. Figures 4 and 5 also demonstrate that at the plotted sparseness levels $s$, the output configuration only slightly influences the sparse model's performance. This can be attributed to the excellent space-filling properties of both sampling algorithms.

It can also be remarked that LOLA-Voronoi performs (slightly) superior to mc-intersite-proj-th, both in terms of $1-r M S E_{M A P}$ and $r M S E_{P O S}$ for a sparsity $s>0.05 \%$ (Fig. 6 provides a zoomed view of 'TOTAL TOT'). As a consequence of its sample generating paradigm, in which the sample density in nonlinear (e.g. with a significant NLOS contribution) regions is augmented, the LOLA-Voronoi algorithm is able to more accurately represent the positioning map. For instance, for $s=0.5 \%$, the mean model resemblance (computed over the 5 output configurations) amounts for mc-intersite-

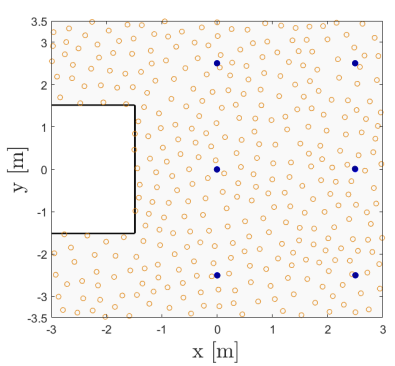

(a)

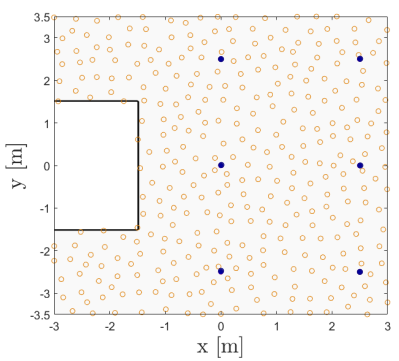

(b)
Fig. 8. Spatial distribution of the sparse model samples for (a) mc-intersiteproj-th and (b) LOLA-Voronoi, blue dots represent the LEDs

proj-th and LOLA-Voronoi to $1-r M S E_{M A P, \text { intersite }}=$ $96.8 \%$ and $1-r M S E_{M A P, L O L A}=97.35 \%$ respectively. LOLA-Voronoi's improved ability for model approximation in $1-r M S E_{M A P}$ also translates in a lower positioning error. The mean positioning rMSE of the 5 output configurations $r M S E_{P O S, \text { intersite }}$ exceeds $r M S E_{P O S, L O L A}$ : e.g. for $s=$ $0.5 \%, r M S E_{P O S, \text { intersite }}=11.18 \mathrm{~cm}$ and $r M S E_{P O S, L O L A}$ $=10.99 \mathrm{~cm}$. In general, 'TOTAL TOT' seems to be the preeminent output configuration. Both LOS and NLOS contributions are taken into account, and the sample selection is only optimised in one dimension which benefits the design time.

The variance of LOLA-Voronoi models are slightly larger than their $m c$-intersite-proj-th counterparts, due to their dependence on the outputs of the selected samples in the early stages of the sequential experimental design. For both sequential design approaches, the modelling variance decreases when more samples are added to the sparse model. At $s>0.2 \%$, the variance is rather limited (upper bounded by about $5 \mathrm{~mm}$ ).

For $s \leq 1 \%$, the uniform grid rMSE (see Fig. 3) $p_{90}=$ $5 \mathrm{~cm}$ still significantly outscores both algorithm's $r M S E_{P O S}$. Part of the performance gap can be attributed to the bilinear interpolation used. Specifically for LOLA-Voronoi, another performance decreasing factor arises due to the LOLA paradigm. For this particular AGV operating area (with Lambertian LEDs), the impact of LOLA can be explained as follows. Per LED, the maximal $I_{P D, i}$ (i.e. the photocurrent contribution) is found in the neighbourhood of the point directly under the LED as a result of $d_{i}$ and $R_{E}\left(\phi_{i}\right)$ being minimal and maximal there respectively (and the NLOS component magnitude being rather limited). For locations around this point of maximal $I_{P D, i}$, the gradient of the $I_{P D, i}$ surface is rather small and the linearity rather large. This is a consequence of the limited magnitude of the gradient of $R_{E}\left(\phi_{i}\right)$ for small $\phi_{i}$. As a result of the LOLA paradigm, the regions of limited gradients are undersampled. This may lead to an underestimation of the maximal $I_{P D, i}$ in the sparse model, which after interpolation may have as an effect that $I_{P D, i}$ (of the interpolated sparse model) may be underestimated in more spatially-spread regions. The influence that non-Lambertian LEDs have on the sparse modelling is therefore worth investigating.

Figure 7 further highlights the difference between $m c$ intersite-proj-th and LOLA-Voronoi-based positioning. It con- 
siders the reference AGV trajectory that is depicted in black. The green and light orange curves denote the localised trajectories of LOLA-Voronoi and mc-intersite-proj-th-based RSS. Here too, the former scores better. For LOLA-Voronoi, the $50^{\text {th }}, 90^{\text {th }}$ and $95^{\text {th }}$ percentile of the rMSE on the positioning estimates amount respectively to $3.7 \mathrm{~mm}, 7.3 \mathrm{~cm}$ and $9.5 \mathrm{~cm}$. The $50^{\text {th }}, 90^{\text {th }}$ and $95^{\text {th }}$ percentile rMSE, corresponding to $m c$-intersite-proj-th equal $3.9 \mathrm{~mm}, 13.8 \mathrm{~cm}$ and $27.3 \mathrm{~cm}$. In comparison, the uniform grid's $p_{50}$ and $p_{90}$ are $0.6 \mathrm{~mm}$ and $1.7 \mathrm{~cm}$. Figure 8 also plots both algorithms' model samples.

Depending on both the required positioning accuracy and the available storage, an appropriate LOLA-Voronoi (or $m c$ intersite-proj-th)-based sparse model can be selected. Imposing a trade-off between $s$ and the guideline $p_{90} \approx 10 \mathrm{~cm}$, the sparse model with $s=0.5 \%$ (with 'TOTAL TOT') is chosen to work with in the following sections. At $s=0.5 \%$, the interpolated sparse model strongly resembles the uniform grid visually. It does show a worsening in positioning rMSE of $6 \mathrm{~cm}$ compared to the uniform grid's. In the subsequent sections, the practicality of this model as a way to compensate for LED ageing will be studied. Technically, for $p_{90} \leq 10 \mathrm{~cm}$ to be satisfied, $s$ should exceed $0.8 \%$.

The outlined sparse modelling approaches can be applied to arbitrary environments (e.g. equipped with different LEDs). They can also be applied in conjunction with different ways of computing the optical propagation (see e.g. the analytical model of Chen et al. [19]). While the SED method remains applicable, the performance of the sparse models will vary though. Furthermore, as SED is an iterative approach, prior (more sparse) or subsequent (less sparse) models can be derived from a current working model.

\section{Fast Model Recomputation for LED Ageing compensation}

Nearing the end of a LED's operating time, the nominal transmitted flux $P_{t, i}$ starts diminishing rapidly (see (1)). Figure 9 illustrates the extent of its impact on the positioning performance. $p_{90, \text { ageing }}, p_{90, \text { ageing, sparse }}$ represent the positioning accuracy in the case of using the uniform grid model and the sparse model (interpolated to the grid) respectively. $t_{o p}$ is set to $50000 \mathrm{~h}$ for each of the LEDs.

From Figure 9, it can be derived that prolonged usage of the same LEDs significantly impacts the positioning performance, once the operating time $t$ exceeds $10000 \mathrm{~h}$. During the first $1000 \mathrm{~h} s$ of operation, the AGV's positioning performance remains unaffected (both $p_{90, \text { ageing }}$ and $p_{90, \text { ageing, sparse }}$ ). The subsequent 2500 working hours $\left(P_{t, i}(3500)=97.5 \% P_{t, i}(0)\right)$ already experience a $p_{90}\left(p_{90}\right.$, ageing $)$ increase to about $10 \mathrm{~cm}$. Operating the same LEDs notably longer violates the $p_{90} \approx$ $10 \mathrm{~cm}$ constraint. Complying with this constraint, requires changing the LEDs or better (certainly cheaper) recomputing the channel model to account for the decreased $P_{t}(t)$. Interestingly, the sparse model is more robust when $t>10000 \mathrm{~h}$ (as it underestimates the channel's DC gain).

$p_{90, \text { sparse recomp. }}$ denotes the $p_{90}-t$ relation, when LED ageing is constantly compensated (e.g. every week) by retriggering the sparse model. Continuous compensation entails estimating the LED ageing, via a (perfect) ageing model or via a closed-loop system (e.g. by placing a PD near each LED to monitor its $P_{t, i}$ and broadcasting this information). Every $5000 \mathrm{hs}$ (infrastructure or receiver-side) recomputing the

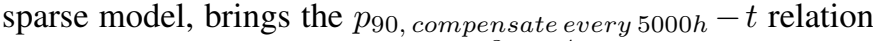
with. Remarkably, during $t \in\left[10^{3}, 10^{4}\right], p_{90, \text { ageing, tot } / L O S}$ (the channel model only accounts for LOS transmission) dips for increasing operating time $t$. In this $t$ region, ageing alleviates (i.e. by attenuating the total induced photocurrent) the positioning error resulting from the by reflections added NLOS photocurrent. The recomputation of the sparse model also supports non-uniform ageing across LEDs.

\section{CONCLUSION}

This paper investigates the aptitude of response adaptive or sequential experimental design in reducing the required storage and computation of the positioning grid's fingerprints in model-fingerprinting-based localisation. It evaluates two iterative, sequential Monte Carlo-based space-filling algorithms: mc-intersite-proj-th and LOLA-Voronoi. Utilising the sequential design methods allows the required storage to drop below 1 percent of the storage that was required for the traditional (oneshot) uniform grid. This is when the sparse model is evaluated on each point of the initial grid. The actual introduceable model sparseness largely depends on the preset requirements in terms of accuracy and storage.

Both sampling algorithms provide a good channel model coverage. For LOLA-Voronoi, the associated propagation model shows, at a $0.5 \%$ sparsity and at an input-referred rms noise current $\sigma$ of $10 \mathrm{nA}$, a $90^{\text {th }}$ percentile positioning rMSE that worsened an acceptable $6 \mathrm{~cm}$ compared to the uniform grid case. The sparse model's performance can be boosted by tailoring the employed interpolation and positioning method (e.g. a different fingerprinting cost function).

Future work will consist of comparing (i.a. in terms of robustness) the sequential design approaches of this paper with other sparse modelling techniques, such as gaussian processbased sampling. Also, the performance of grid-based SED will be compared to the non-grid based SED of this paper. The $m c$ intersite-proj-th and LOLA-Voronoi algorithms will be required to only output samples laying on the uniform positioning grid.

In addition, the introduction of sparsity in the channel models of vast positioning halls will be evaluated. Lastly, the experimental verification of the conclusions of this paper is part of the future work.

\section{ACKNOWLEDGMENT}

This work was performed within LEDsTrack, a project that is co-financed by imec and that is receiving project support from Flanders Innovation \& Entrepreneurship.

\section{REFERENCES}

[1] Grand View Research, "Automated Guided Vehicle Market Size, Share \& Trends Analysis Report By Navigation Technology (Magnetic, Laser, Vision-guided), By Type, By Application, By End-use, By Region, And Segment Forecasts, 2018 - 2024," GVR-1-68038-153-5, 2018, pp. 140. 


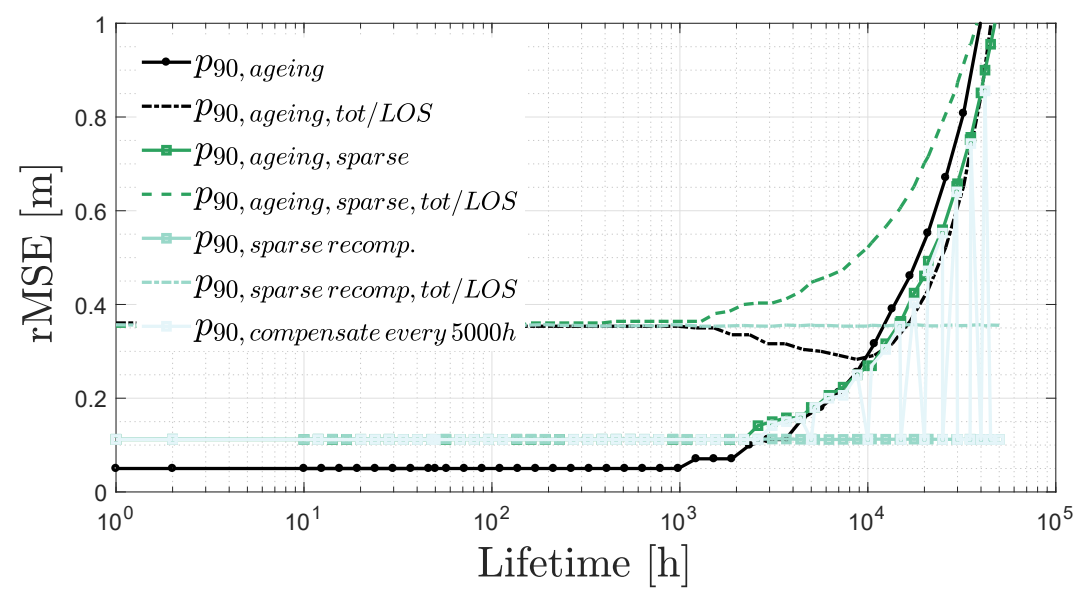

Fig. 9. The impact of a sweeping LED operating time and corresponding LED ageing on the $p_{90}$ for $\sigma=10 \mathrm{nA}$

[2] European Commission, "Traceability across the Value Chain, Advanced tracking systems," Business Innovation Observatory Contract, no. 190/PP/ENT/CIP/12/C/N03C01, 2015.

[3] R. G. Yudanto and F. Petr, "Sensor Fusion for Indoor Navigation and Tracking of Automated Guided Vehicles," International Conference on Indoor Positioning and Indoor Navigation (IPIN), 2015, pp 1-8.

[4] Z. Zhou, "Indoor positioning algorithm using light-emitting diode visible light communications," Optical Engineering, vol. 51, no. 8, 2012, pp 6.

[5] L. Li, P. Hu, C. Peng, G. Shen and F. Zhao, "Epsilon: A Visible Light Based Positioning System," 11th USENIX Symposium on Networked Systems Design and Implementation (NSDI), 2014, pp. 331-343.

[6] Y. S. Kuo, P. Pannuto, K. J. Hsiao and P. Dutta, "Luxapose: Indoor Positioning with Mobile Phones and Visible Light," International Conference on Mobile Computing and Networking (MobiCom), 2014, pp 299-301.

[7] Y. Hu, Y. Xiong, W. Huang, X. Y. Li, Y. Zhang, X. Mao, P. Yang and C. Wang, "A Visible Light Based Indoor Positioning System, International Conference on Distributed Computing Systems (ICDCS), 2015, pp $1-16$.

[8] J. Luo, L. Fan and L. Husheng, "Indoor Positioning Systems Based on Visible Light Communication: State of the Art," IEEE Communications Surveys \& Tutorials, vol. 19, no. 4, 2017, pp. 2871-2893.

[9] J. Armstrong, Y. A. Sekercioglu and A. Neild, "Visible Light Positioning: A Roadmap for International Standardization," IEEE Communications Magazine, vol. 51, no. 12, 2013, pp. 68-73.

[10] J. L. Gardner and F. J. Wilkinson, "Angular effects in silicon photodiode responsivity comparisons," Metrologia, vol. 34, 1997, pp. 111-114.

[11] T. Saito, "Spectral Properties of Semiconductor Photodiodes, Advances in Photodiodes," IntechOpen, (2018, Mar. 12), Available: https://www.intechopen.com/books/advances-in-photodiodes/ spectral-properties-of-semiconductor-photodiodes.

[12] D. Plets, A. Eryildirim, S. Bastiaens, N. Stevens, L. Martens and W. Joseph, "A Performance Comparison of Different Cost Functions for RSS-Based Visible Light Positioning Under the Presence of Reflections," Proceedings of the 4th ACM Workshop on Visible Light Communication Systems (VLCS), 2017, pp. 37-41.

[13] K. Crombecq, L. De Tommasi, D. Gorissen and T. Dhaene, "A novel sequential design strategy for global surrogate modeling," Proceedings of the 2009 Winter Simulation Conference (WSC), 2009, pp. 731-742.

[14] K. Crombecq, E. Laermans and T. Dhaene, "Efficient space-filling and non-collapsing sequential design strategies for simulation-based modeling," European Journal of Operational Research, vol. 214, no. 3, 2011, pp. 683-696.

[15] K. Crombecq, "Surrogate Modelling of Computer Experiments with Sequential Experimental Design", Ph.D. dissertation, CoMP, Universiteit Antwerpen, Antwerp, IBCN, Universiteit Gent, Ghent, 2011.

[16] E. M. Jeong, S. H. Yang, H. S. Kim and S. K. Han, "Tilted receiver angle error compensated indoor positioning system based on visible light communication," Electronics Letters, vol. 49, no. 14, 2013, pp. 2.

[17] N. A. Mohammmed and M. A. Elkarim, "Exploring the effect of diffuse reflection on indoor localization systems based on RSSI-VLC," Optics Express, vol. 23, no. 16, 2015, pp. 20297-20313.
[18] W. Gu, M. Aminikashani, P. Deng and M. Kavehrad, "Impact of Multipath Reflections on the Performance of Indoor Visible Light Positioning Systems," Journal of Lightwave Technology, vol. 34, no. 10, 2016, pp 2578-2587.

[19] C. Chen, D. A. Basnayaka, X. Wu and H. Haas, "Efficient Analytical Calculation of Non-Line-of-Sight Channel Impulse Response in Visible Light Communications," Journal of Lightwave Technology, vol. 36, no. 9, 2018, pp. 1666-1682.

[20] C. Liu, A. Kiring, N. Salman, L. Mihaylova and I. Esnaola, "A Kriging algorithm for location fingerprinting based on received signal strength," Sensor Data Fusion: Trends, Solutions, Applications (SDF), 2015, pp. $1-6$

[21] K. Chen, Y. Mi, Y. Shen, Y. Hong, A. Chen and M. Lu, "SparseLoc: Indoor Localization Using Sparse Representation," IEEE Access, vol. 5, pp. 20171-20182, 2017.

[22] S. Aerts, D. Deschrijver, W. Joseph, L. Verloock, F. Goeminne, L. Martens and T. Dhaene, "Exposure assessment of mobile phone base station radiation in an outdoor environment using sequential surrogate modeling," Bioelectromagnetics, vol. 34, no. 4, 2013, pp. 300-311.

[23] H. Steendam, "A 3-D Positioning Algorithm for AOA-Based VLP With an Aperture-Based Receiver," IEEE Journal on Selected Areas in Communications, vol. 36, no. 1, 2018, pp. 23-33.

[24] S. De Lausnay, L. De Strycker, J. P. Goemaere, N. Stevens and B. Nauwelaers, "A Visible Light Positioning system using Frequency Division Multiple Access with square waves," International Conference on Signal Processing and Communication Systems (ICSPCS), 2015, pp. $1-7$

[25] W. D. van Driel and X. Fan, 'Solid State Lighting Reliability, Components to Systems“,” Springer-Verlag New York, 2013, pp. 618.

[26] P. Lall, J. Wei and P. Sakalaukus, "Life prediction and classification of failure modes in solid state luminaires using Bayesian Probabilistic Models," 64th Electronic Components and Technology Conference (ECTC), 2014, pp. 2053-2062.

[27] M. Wagner, A. Herzog, H. Ganev and T. Q. Khanh, "LED aging acceleration An analysis from measuring and aging data of 14,000 hours LED degradation," 12th China International Forum on Solid State Lighting (SSLCHINA), 2015, pp. 75-78.

[28] M. Uysal, F. Miramirkhani, O. Narmanlioglu, T. Baykas and E. Panayirci, "IEEE 802.15.7r1 Reference Channel Models for Visible Light Communications," IEEE Communications Magazine, vol. 55, no. 1, 2017, pp. 212-217.

[29] J. M. Kahn and J. R. Barry, "Wireless infrared communications," Proceedings of the IEEE, vol. 85, no. 2, 1997, pp. 265-298.

[30] C. R. Lomba, R. T. Valadas and A. M. de Oliveira Duarte, "Experimental characterisation and modelling of the reflection of infrared signals on indoor surfaces," IEE Proceedings - Optoelectronics, vol. 145, no. 3, 1998, pp. 191-197.

[31] M. L. Marceau and M. G. VanGeem, "Solar Reflectance Values of Concrete," (2018, Mar. 12), Available: http://www.cement.org/docs/ default-source/fc_concrete_technology/sn2982a-solar-reflectance-valuesof-concrete.pdf. 